\title{
Same role but different actors: genetic regulation of post-translational modification of two distinct proteins
}

Arianna Landini ${ }^{* 1}$, Irena Trbojević-Akmačić $*^{2}$, Pau Navarro ${ }^{3}$, Yakov A. Tsepilov ${ }^{4,5}$, Sodbo Z. Sharapov ${ }^{4}$, Frano Vučković ${ }^{2}$, Ozren Polašek ${ }^{6,7}$ Caroline Hayward $^{3}$, Tea Petrovic ${ }^{2}$, Marija Vilaj $^{2}$, Yurii S. Aulchenko ${ }^{4}$, Gordan Lauc ${ }^{2,8}$, James F. Wilson*1,3 ${ }^{{ }^{1,3}}$ Lucija Klarić ${ }^{* 3}$

1 Centre for Global Health Research, Usher Institute, University of Edinburgh, Edinburgh, United Kingdom

2 Genos Glycoscience Research Laboratory, Zagreb, Croatia

3 MRC Human Genetics Unit, Institute for Genetics and Cancer, University of Edinburgh, Edinburgh, United Kingdom

4 Laboratory of Glycogenomics, Institute of Cytology and Genetics, Novosibirsk, Russia

5 Laboratory of Theoretical and Applied Functional Genomics, Novosibirsk State University, Novosibirsk, Russia

6 Department of Public Health, School of Medicine, University of Split, Split, Croatia

7 Gen-info Ltd., Zagreb, Croatia

8 Faculty of Pharmacy and Biochemistry, University of Zagreb, Zagreb, Croatia

* Authors contributed equally. Correspondence to: J.F.W (jim.wilson@ed.ac.uk) or L.K. (lucija.klaric@ed.ac.uk)

Post-translational modifications (PTMs) diversify protein functions and dynamically coordinate their signalling networks, influencing most aspects of cell physiology. Nevertheless, their genetic regulation or influence on complex traits is not fully understood. Here, we compare for the first time the genetic regulation of the same PTM of two proteins - glycosylation of transferrin and immunoglobulin G (IgG). By performing genome-wide association analysis of transferrin glycosylation, we identified 10 significantly associated loci, all novel. Comparing these with IgG glycosylationassociated genes, we note protein-specific associations with genes encoding glycosylation enzymes (transferrin - MGAT5, ST3GALA, B3GAT1; IgG - MGAT3, ST6GAL1) as well as shared associations (FUT6, FUT8). Colocalisation analyses of the latter suggest that different causal variants in the FUT genes regulate fucosylation of the two proteins. We propose that they affect the binding of different transcription factors in different tissues, with fucosylation of IgG being regulated by IKZF1 in B-cells and of transferrin by HNF1A in liver. 


\section{Introduction}

Post-translational modifications (PTMs) are essential mechanisms used by cells to diversify and extend their protein functions beyond what is dictated by protein-coding sequences in the genome. These chemical reactions range from the addition of small moieties, such as phosphate (phosphorylation), complex biomolecules, as in glycosylation, to proteolytic cleavage $^{1}$. PTMs alter the structure and properties of proteins and are thus involved in the dynamic regulation of most cellular events. It is common for a PTM enzyme to target multiple substrates or interact with multiple sites. For example, only 18 histone deacetylases target more than 3600 acetylation sites on 1750 proteins $^{2}$. Environmental or pathological conditions can lead to dysregulation of PTM activities, which has been related to aging ${ }^{3}$ and several diseases, including cancer, diabetes, and neurodegeneration ${ }^{4-10}$. Despite their importance, little is known about genetic regulation of post-translational modifications.

$\mathrm{N}$-glycosylation is one of the most common protein PTMs, where carbohydrate structures called glycans are covalently attached to an asparagine (Asn) residue of a polypeptide backbone. N-glycans are characterised by vast structural diversity and high complexity. While polypeptides are encoded by a single gene, $\mathrm{N}$-glycan structures result from a sophisticated interplay of glycosyltransferases, glycosidases, transporters, transcription factors, and other proteins ${ }^{11}$. Protein $\mathrm{N}$-glycosylation is involved in a multitude of biological processes $^{12}$. Accordingly, changes in $\mathrm{N}$-glycosylation patterns have been associated with aging $^{13}$ and a wide range of diseases, including Parkinson's disease ${ }^{14}$, lower back pain ${ }^{15}$, rheumatoid arthritis ${ }^{16}$, ulcerative colitis ${ }^{17}$, Crohn's disease ${ }^{17}$, type 2 diabetes ${ }^{18}$ and cancer ${ }^{19-21}$. In addition, $\mathrm{N}$-glycans are considered as potential therapeutic targets ${ }^{22}$ and prognostic biological markers ${ }^{18,23-25}$.

As with other PTMs, genetic regulation of N-glycosylation is not yet fully understood. Previous genome-wide association studies (GWAS) have so far focused either on the Nglycome of total blood plasma proteins as a whole or on glycosylation of one specific protein - immunoglobulin $\mathrm{G}(\mathrm{IgG})^{26-33}$. IgG antibodies are one of the most abundant proteins in human serum, and their alternative $\mathrm{N}$-glycosylation is suggested to trigger different immune response and thus impacts the action of the immune system $^{34}$. N-glycan structures are predominantly of the biantennary complex type and vary due to additions of core fucose, galactose, sialic acid, and bisecting $\mathrm{N}$-acetylglucosamine (GlcNAc), with disialylated digalactosylated biantennary glycan with core fucose and bisecting GlcNAc being the most complex $\mathrm{N}$-glycan structure on $\mathrm{IgG}^{35}$. While a clear overlap in genetic control between total plasma proteins and IgG N-glycosylation was highlighted by previous studies ${ }^{28}$, it was not possible, until now, to identify protein-specific N-glycosylation pathways for glycoproteins other than IgG due to technical challenges hampering isolation of other glycoproteins in large cohorts.

Here we report genes associated with the regulation of transferrin $\mathrm{N}$-glycosylation and compare these with the genetic regulation of glycosylation of a different protein ( $\mathrm{IgG}$ ). Transferrins are blood plasma glycoproteins regulating the level of iron in an organism. Iron plays a central role in many essential biochemical processes of human physiology: the cells' need for iron in the face of potential danger as an oxidant has given rise to a complex system 
that tightly regulates iron levels, tissue distribution, and bioavailability ${ }^{36}$. Human transferrin has two N-glycosylation sites - at the N432 and N630 residues, with biantennary disialylated digalactosylated glycan structure without fucose being the most abundant glycan attached $^{37,38}$. We performed, for the first time, genome-wide association meta-analysis (GWAMA) of 35 transferrin N-glycan traits $(\mathrm{N}=1890)$ and compared it with GWAMA of 24 IgG N-glycan traits $(\mathrm{N}=2020)$ in European-descent cohorts, discovering both protein-specific and shared associations. For loci associated with the N-glycosylation PTM of both transferrin and $\mathrm{IgG}$, we used colocalisation analysis to assess whether the underlying causal variants are protein-specific or rather shared between these proteins. We then suggested a molecular mechanism by which these independent causal variants could regulate the expression of glycosylation related genes in different tissues. To the best of our knowledge, this is the first study investigating whether the same PTM of two proteins is regulated by the same genes and whether they are driven by the same causal genetic variants.

\section{$\underline{\text { Results }}$}

\section{Loci associated with transferrin N-glycosylation}

To investigate the genetic control of transferrin N-glycosylation and assess whether the same genes and underlying causal variants are associated with $\mathrm{N}$-glycosylation of both transferrin and $\mathrm{IgG}$, we first performed GWAS of glycosylation for each protein (i.e. transferrin and $\mathrm{IgG}$ ). A more extensive GWAS on the genetic regulation of $\mathrm{IgG}$ glycosylation has already been published ${ }^{30}$, so we focus here on glycosylation of transferrin. We performed GWAS of 35 ultra-high-performance liquid chromatography (UHPLC)-measured transferrin N-glycan traits and Haplotype Reference Consortium (HRC) r1.1-imputed genetic data in two cohorts of European descent $(\mathrm{N}=1890)$. To identify secondary association signals at each genomic region, we performed approximate conditional analysis on transferrin $\mathrm{N}$-glycan traits using GCTA-COJO software ${ }^{39}$. Overall, we identified 26 independently contributing variants, located in 10 genomic loci significantly associated ( $\mathrm{p}$-value $\leq 1.43 \times 10^{-9}$, Bonferroni adjusted for the number of glycan traits) with at least one of the 35 transferrin N-glycan traits (Table 1, Figure 1, complete list of all associations in Supplementary Table 1). Multiple SNPs independently contributed to transferrin $\mathrm{N}$-glycans variation in 6 out of 10 loci, all mapping to glycosyltransferase genes, plus the transferrin $(T F)$ gene. The highest number of independently associated SNPs (7) was observed for the sialyltransferase locus, ST3GALA, followed by 4 SNPs in the acetylglucosaminyltransferase locus, MGAT5, and in the glucuronyltransferase locus, B3GAT1. Lastly, 2 SNPs independently contributed to transferrin glycosylation in the fucosyltransferase loci, FUT8 and FUT6, and also the transferrin $(T F)$ locus itself (Supplementary Table 2). 
bioRxiv preprint doi: https://doi.org/10.1101/2021.05.04.442584; this version posted May 4, 2021. The copyright holder for this preprint (which was not certified by peer review) is the author/funder, who has granted bioRxiv a license to display the preprint in perpetuity. It is made available under aCC-BY-ND 4.0 International license.

Table 1. Loci genome-wide significantly associated with at least one of the 35 transferrin N-glycan traits in GWAMA. Glycosyltransferase loci are reported at the top of the table, while other loci are listed at the bottom of the table. Each locus is represented by the SNP with the strongest association in the region.

\begin{tabular}{|c|c|c|c|c|c|c|c|c|c|c|c|c|}
\hline Locus & Gene & SNP & EA & $\mathbf{O A}$ & EAF & $\begin{array}{l}\text { No. of } \\
\text { SNPs }\end{array}$ & $\begin{array}{c}\text { Lead } \\
\text { glycan }\end{array}$ & $\begin{array}{l}\text { Phe. } \\
\text { var. }\end{array}$ & $\begin{array}{c}\text { No. of } \\
\text { glycans }\end{array}$ & Beta & SE & $\mathbf{P}$ \\
\hline $\begin{array}{c}2: 134839539- \\
135024803\end{array}$ & MGAT5 & rs2442046 & $\mathrm{C}$ & G & 0.747 & 4 & TfGP23 & 0.071 & 4 & -0.44 & 0.037 & $1.38 \times 10^{-32}$ \\
\hline $\begin{array}{l}11: 12605298 \\
8-126312874\end{array}$ & ST3GALA & rs4055121 & $\mathrm{T}$ & $\mathrm{C}$ & 0.12 & 7 & TfGP17 & 0.131 & 9 & 0.782 & 0.046 & $9.67 \times 10^{-64}$ \\
\hline $\begin{array}{c}14: 65751627- \\
66281192\end{array}$ & FUT8 & rs2411815 & A & $\mathrm{T}$ & 0.306 & 2 & TfGP20 & 0.092 & 3 & -0.469 & 0.035 & $2.69 \times 10^{-41}$ \\
\hline $\begin{array}{c}19: 5813766- \\
5841356\end{array}$ & FUT6 & rs12019136 & A & G & 0.039 & 2 & TfGP32 & 0.079 & 5 & -1.016 & 0.083 & $2.00 \times 10^{-34}$ \\
\hline $\begin{array}{c}5: 169535155- \\
169535155\end{array}$ & FOXI1 & $\begin{array}{c}\text { rs11539930 } \\
7\end{array}$ & $\mathrm{~T}$ & $\mathrm{C}$ & 0.018 & 1 & TfGP23 & 0.031 & 1 & 0.941 & 0.152 & $5.18 \times 10^{-10}$ \\
\hline $\begin{array}{c}8: 15831868- \\
16623073\end{array}$ & MSR1 & rs41341748 & A & G & 0.027 & 1 & TfGP35 & 0.031 & 1 & 0.778 & 0.109 & $1.16 \times 10^{-12}$ \\
\hline $\begin{array}{l}11: 11438144 \\
8-114384985\end{array}$ & $\begin{array}{l}\text { NXPE1/ } \\
\text { NXPE4 }\end{array}$ & rs1671819 & A & G & 0.454 & 1 & TfGP14 & 0.02 & 1 & -0.2 & 0.032 & $3.32 \times 10^{-10}$ \\
\hline $\begin{array}{l}12: 12142026 \\
3-121424861\end{array}$ & HNF1A & rs2393775 & A & G & 0.638 & 1 & TfGP28 & 0.019 & 1 & -0.203 & 0.033 & $8.97 \times 10^{-10}$ \\
\hline
\end{tabular}

Locus - coded as "chromosome: locus start-locus end" (GRCh37 human genome build); Gene - suggested candidate gene; SNP - variant with the strongest association in the locus; EA - SNP allele for which the effect estimate is reported; OA - other allele; EAF - frequency of the effect allele; No. of SNPs - number of SNPs in the locus independently contributing to trait variation according to GCTA-COJO; Lead glycan - glycan trait with the strongest association to the reported SNP; Phe. var. - proportion of variance in phenotype explained by the strongest associated SNP; No. of glycans - number of glycan traits significantly associated with variants at the given locus; Beta - effect estimate for the SNP and glycan with the strongest association in the locus; SE - standard error of the effect estimate, $\mathrm{P}$ - p-value of the effect estimate. 


\section{Prioritising candidate genes associated with transferrin N-glycosylation}

For the 10 loci associated with the transferrin N-glycome, we identified plausible candidate genes following multiple lines of evidence, such as evaluating the biological role of the candidate gene in the context of protein N-glycosylation, assessing SNP pleiotropy with eQTLs, and investigating variant effects on the coding sequence or on putative transcription factor binding sites.

Positional mapping and biological role. The majority of genes that were closest to transferrin N-glycosylation-associated variants had a clear biological link to protein Nglycosylation. In particular, for 5 out of 10 loci the closest genes (i.e. MGAT5, ST3GAL4, B3GAT1, FUT8, and FUT6) encode glycosyltransferases, key enzymes in protein glycosylation, that have been previously associated with $\mathrm{IgG}$ and/or total plasma protein glycosylation (Supplementary Table 3). Another gene closest to transferrin N-glycosylationassociated variants and with a validated functional role in plasma proteins glycosylation is HNF1A, a transcription factor previously associated with protein fucosylation (Supplementary Table 3). On the other hand, we also identified 3 loci that had not been associated with $\mathrm{N}$-glycosylation. A locus on chromosome 3 contains the transferrin $(T F)$ gene, which encodes the transferrin glycoprotein. A locus on chromosome 5 contains FOXI1, encoding a member of the forkhead family of transcription factors (Forkhead box I1). Finally, a locus on chromosome 8 contains the MSRl gene, encoding the class A macrophage scavenger receptor, a trimeric integral membrane glycoprotein. Another gene of potential biological relevance at the chromosome 8 locus is the tumour suppressor candidate 3 (TUSC3), which encodes a protein localised to the endoplasmic reticulum and acting as a component of the oligosaccharyltransferase complex, responsible for $\mathrm{N}$-linked protein glycosylation.

Overlap and colocalisation with eQTL. Using eQTL analysis in PhenoScanner, transferrin $\mathrm{N}$-glycan-associated genetic variants (and their proxies, LD $\mathrm{r}^{2}>0.8$ ) were identified to be significantly associated with the expression of multiple genes in several human tissues involved in transferrin metabolism (Supplementary Table 4a). For example, transferrin glycosylation variants were associated with ST3GALA expression in liver and whole blood, with B3GAT1 expression in visceral adipose omentum, liver, and whole blood, with $T F$ expression in several adipose tissues and with HNF1A, FUT8, and MGAT5 expression in whole blood. The majority of these genes were also the closest to the strongest association in the locus. We next used Summary data-based Mendelian Randomization (SMR) analysis followed by the Heterogeneity in Dependent Instruments (HEIDI) test ${ }^{41}$ to assess whether expression of these genes colocalises with transferrin glycosylation (TfGP) traits. SMRHEIDI provided evidence of pleiotropy, suggesting that the same underlying causal SNPs are likely to regulate both transferrin glycosylation traits and gene expression, for B3GAT1 in liver and peripheral blood and ST3GAL4 in liver (Supplementary Table 4b). 
Analysis of possible effects on amino acid sequence. We next explored whether any of the SNPs independently contributing to transferrin glycosylation (or their proxies) result in a change of amino acid sequence using the Ensembl Variant Effect Predictor (VEP) ${ }^{42}$. While the majority of associated variants $(>60 \%)$ were classified as intronic, several SNPs were identified as missense variants: rs115399307 (5_169535155_T/C) causes the substitution of the non-polar, aliphatic amino acid isoleucine (I) to the polar, hydrophilic amino acid threonine (T) in the FOXI1 transcription factor. Similarly, NXPE4 variant rs550897 (11_114442103_A/G, $\mathrm{r}^{2}=0.94$ with rs1671819) causes an amino acid substitution from tyrosine (Y) to histidine (H), while FUT6 variant rs 17855739 (19_5831840_T/C, $\mathrm{r}^{2}=0.95$ with rs12019136) encodes a change from negatively charged glutamic acid (E) to positively charged lysine $(\mathrm{K})$, which leads to a full-length, but inactive, enzyme ${ }^{43}$. Genetic variant rs41341748 (8_16012594_A/G) disrupts a stop codon sequence in MSR1, causing an elongated transcript with the amino acid arginine (Arg) added to the protein chain (Supplementary Table 5).

Analysis of possible effects on transcription factor binding sites. Finally, we used the regulatory sequence analysis tools $(\mathrm{RSAT})^{44}$ to assess if transferrin N-glycosylationassociated genetic variants overlap transcription factor-binding sites (TFBSs) and are likely to affect transcription factor (TF) binding. From the list of prioritised genes, we selected the two encoding transcription factors, FOXI1 and HNF1A, and checked whether associated variants in the remaining 8 loci were likely to affect their binding. Overall, binding of both FOXI1 and HNF1A transcription factors is likely to be affected by the sentinel variant (the SNP with lowest $\mathrm{p}$-value in the region for the given glycan trait) in the FUT8 gene. In addition, binding of HNF1A is likely to be affected also by the sentinel variants in the $T F$ and ST3GAL4 loci (Supplementary Table 6).

\section{$\underline{\text { Shared genetic associations with complex traits and diseases }}$}

To assess whether transferrin glycosylation variants were also associated with complex traits and diseases we used PhenoScanner ${ }^{45}$, followed by SMR-HEIDI to determine whether the shared associations are caused by the same underlying causal variant (pleiotropy). We observed an overlap of transferrin N-glycan-associated SNPs and their proxies with variants associated with complex trait- and disease-associated variants for 5 out of 10 glycosylation loci (Supplementary Table 7a). Glycosylation SNPs at the NXPE1/NXPE4 locus were pleiotropic with ulcerative colitis, and those from the HNF1A locus with C-reactive protein levels, LDL and total cholesterol (Supplementary Table 7b). For the remaining shared associations, we had no power to assess pleiotropy (Supplementary Results for further details). Interestingly, variants at the $T F$ locus have been previously associated with serum concentration of carbohydrate-deficient transferrins (CDT) (Supplementary Table 7a), less glycosylated transferrin isoforms traditionally used as a biomarker of excessive alcohol consumption $^{46}$, thus corroborating our finding for a related trait. 


\section{Comparison of genetic regulation of glycosylation of transferrin and immunoglobulin G}

One of the main aims of this study is to understand if the N-glycosylation of two proteins is regulated by the same enzymes and if so, whether the same underlying genetic variant or a set of variants are driving the process. To address this question, in addition to the already described GWAMA of transferrin glycosylation, we performed a GWAMA of 24 UHPLC IgG N-glycan traits in the same individuals $(\mathrm{N}=2020)$, following the same protocol. 13 loci were significantly associated with at least one of the 24 IgG N-glycan traits (Figure 1, Supplementary Table 8). The IgG N-glycome GWAS was annotated using genes or sets of genes prioritised by Klarić et al. ${ }^{30}$ By comparing the two GWAS we discovered mainly protein-specific associations, but also two genomic regions that were associated with glycosylation of both proteins (Figure 1). The protein-specific associations were with genes encoding known glycosylation enzymes (transferrin - MGAT5, ST3GALA, B3GAT1; IgG ST6GAL1, MGAT3), but also with transcription factors (transferrin - HNF1A, FOXI1; IgG $I K Z F 1, R U N X 3$ ), the protein itself (transferrin - TF; IgG - TMEM121, gene in proximity of $I G H$ genes encoding immunoglobulin heavy chains) as well as other genes (transferrin MSR1; IgG - TXLNB, ABCF2, SMARCB1 region, HLA-region). Interestingly, the regions containing FUT8 and FUT6, genes encoding fucosyltransferases, enzymes adding core and antennary fucose, respectively, to the synthetized glycan, were associated with glycosylation of both proteins (Figure 1). We then proceeded to assess whether the same underlying causal variants in these regions are controlling the process for both proteins using colocalisation analysis.

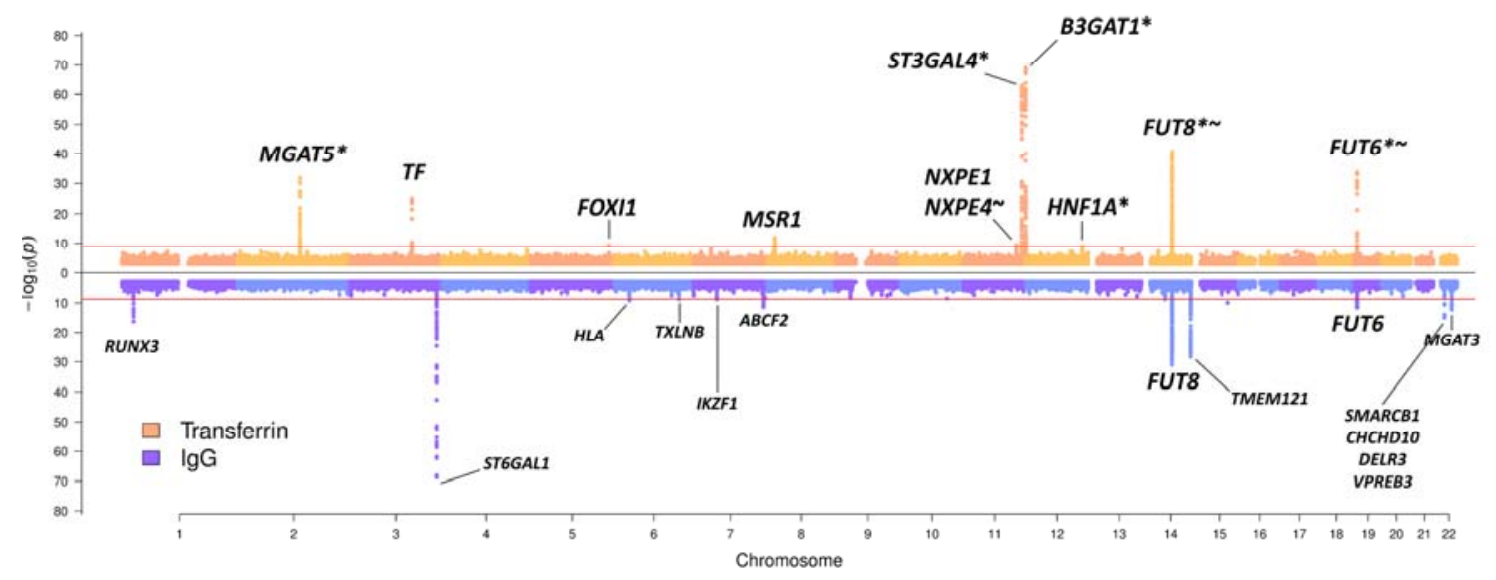

Figure 1. Transferrin and IgG N-glycome GWAMA summary Miami plot. Miami plot pooling together meta-analysis results obtained across all 35 transferrin glycan traits at the top in orange, and across all $24 \mathrm{IgG}$ glycan traits at the bottom in blue. For transferrin N- 
glycome associations, * marks loci previously reported in total blood plasma N-glycome GWAS $^{26-28}$, while $\sim$ marks loci previously reported in IgG N-glycome GWAS ${ }^{30-32,40}$. For simplicity, SNPs with p-value $>1 \times 10^{-3}$ are not plotted. The Bonferroni-corrected genomewide significance threshold for the transferrin $\mathrm{N}$-glycome meta-analysis (horizontal red line in the top part of the plot) corresponds to $1.43 \times 10^{-9}$, while the Bonferroni-corrected genomewide significance threshold for the IgG N-glycome meta-analysis (horizontal red line in the bottom part of the plot) corresponds to $2.08 \times 10^{-9}$. Gene or sets of genes annotated for transferrin N-glycome loci have been prioritised in this study; gene or sets of genes annotated for IgG N-glycome loci are those prioritised by Klarić et al. ${ }^{30}$

Given that multiple glycan traits of the same protein can be associated with the same locus, we first asked whether all glycan traits of the same protein associated with a certain locus, colocalise (Supplementary Figure 1). Indeed, we found strong support for colocalisation (PP.H4 > $80 \%$, where PP.H4 represents the posterior probability for the same underlying causal variant contributing to trait variation), suggesting that for a given protein, all glycan traits associated with these loci are regulated by the same underlying causal variant (Supplementary Table 9, Supplementary Figure 2-4). One example of within-protein colocalisation can be seen in Figure 2. We next tested whether at the same genomic region, glycosylation of two different proteins is regulated by the same underlying causal variants. For this, we selected as the protein-representative glycan trait the one with the lowest p-value in the given region (one pair for each locus - transferrin TfGP20 and IgG GP7 for the FUT8 locus and transferrin TfGP32 and IgG GP20 for the FUT6 locus) and proceeded to test for colocalisation between glycosylation of the two proteins. We found strong support against colocalisation in both genomic regions (PP.H3 $=100 \%$ at FUT8 locus, PP.H3 $=99.71 \%$ at FUT6 locus, where PP.H3 represents the posterior probability for different underlying causal variants contributing to trait variation) (Figure 3 and Figure 4, Supplementary Table 10). Since colocalisation methods are sensitive to multiple independent variants in the region contributing to the trait variation, which was the case here, we validated our findings with the PwCoCo approach ${ }^{47}$ (Methods) and again, obtained robust evidence against the colocalisation hypothesis for all tested traits in both loci (Supplementary Table 10 and Supplementary Results for further details). 
A)

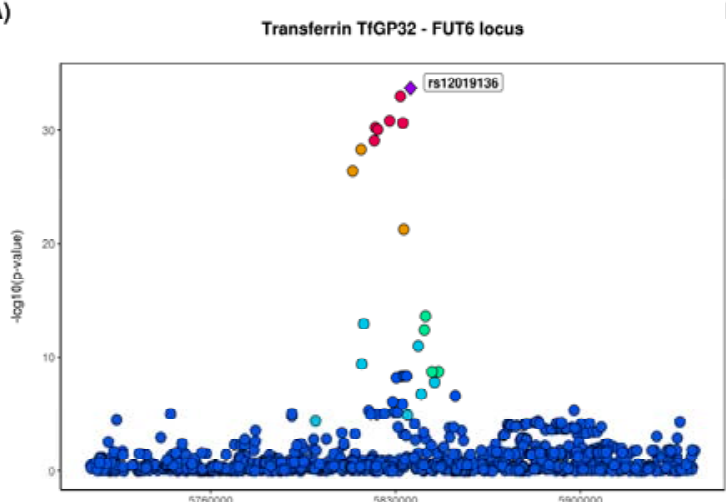

B)

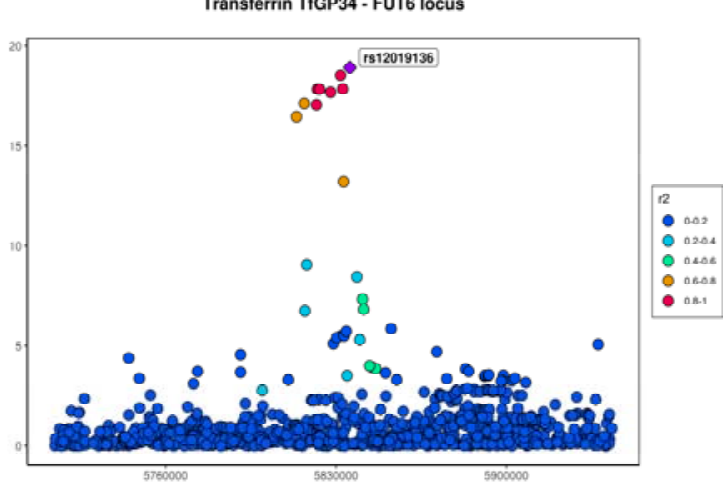

C)

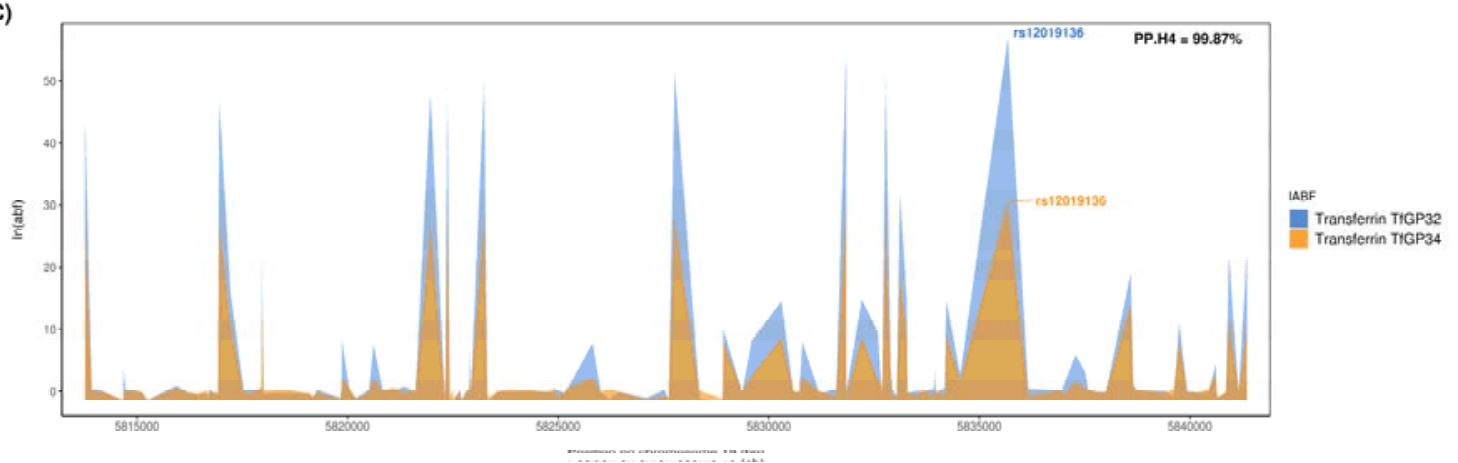

Figure 2. Local association patterns for transferrin (A) TfGP32 and (B) TfGP34 glycans, and (C) their colocalisation pattern at the FUT6 locus. TfGP32 and TfGP34 association patterns colocalise, with PP.H4 (posterior probability for hypothesis 4, of colocalisation) of $99.87 \%$. c) The logarithm of Approximate Bayes Factor (ABF) of each SNP for transferrin TfGP32 and transferrin TfGP34 in the FUT6 region shows that TfGP32 and TfGP34 associations are concordant (the patterns of $\ln (\mathrm{ABF})$ calculated for each SNP of both traits overlap), suggesting that the same underlying causal variant is associated with both traits.

A)

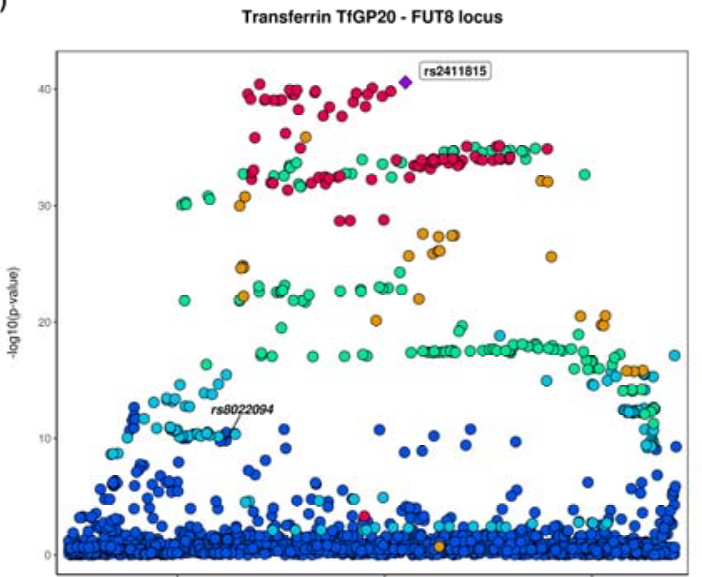

B)

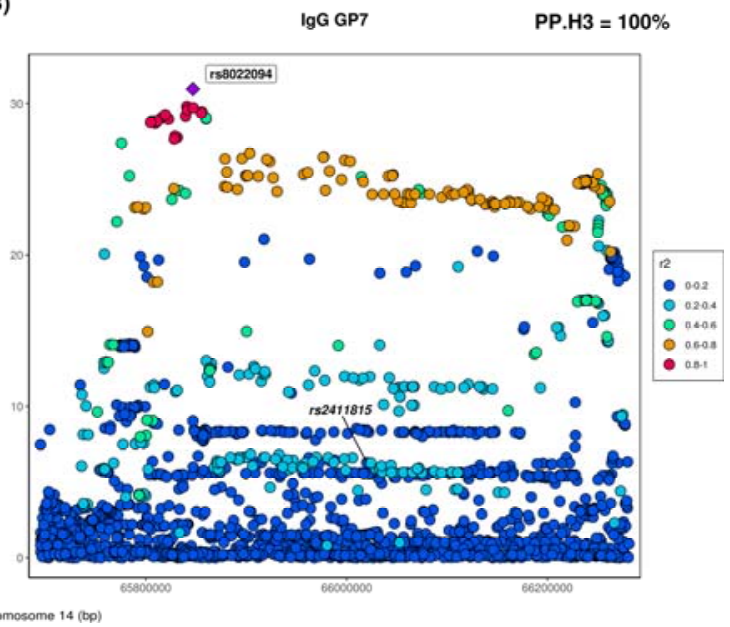


Figure 3. Local association patterns for (A) transferrin TfGP20 and (B) IgG GP7 glycans at the FUT8 locus. TfGP20 and IgG GP7 association patterns do not colocalise (PP.H3 = $100 \%$ - posterior probability for hypothesis 3, of different causal variants). Colocalisation patterns are not reported since the width of the FUT8 region makes the plot non-informative.

A)

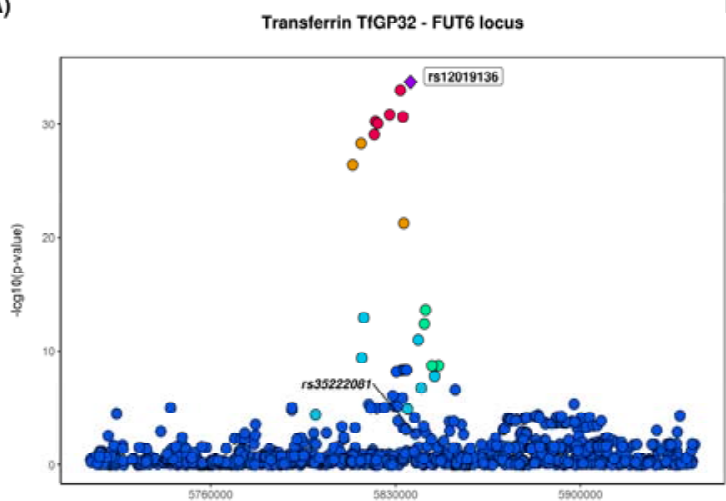

B)

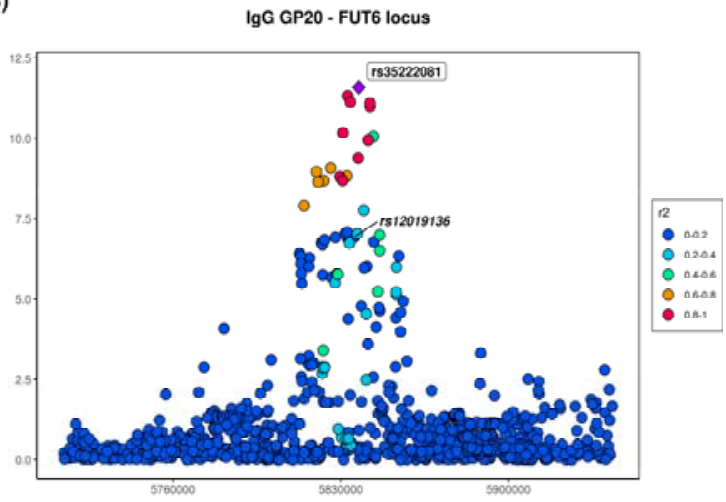

C)

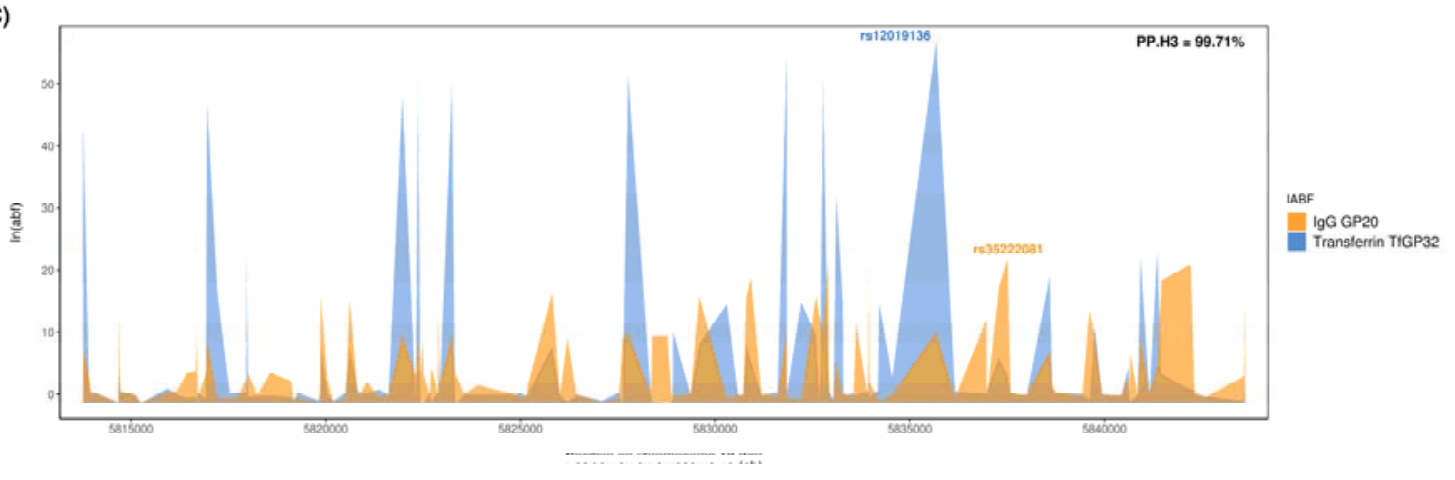

Figure 4. Local association patterns for (A) transferrin TfGP32 and (B) IgG GP20 glycans, and (C) their colocalisation pattern at the FUT6 locus. TfGP32 and IgG GP20 association patterns do not colocalise (PP.H3, posterior probability for hypothesis 3, of different causal variants $=99.7 \%)$. The logarithm of Approximate Bayes Factor $(A B F)$ of each SNP for transferrin TfGP32 and IgG GP20 in the FUT6 region shows that TfGP32 and GP20 associations are not concordant (the patterns of $\ln (\mathrm{ABF})$ calculated for each SNP of both traits do not overlap), suggesting that two different underlying causal variants in this region regulate glycosylation of these two proteins.

Having established that different underlying causal variants regulate glycosylation at the FUT6 and FUT8 loci, we next explored the potential mechanisms behind these associations. The sentinel transferrin glycosylation SNP in the FUT8 region is likely to affect binding of 
the HNF1A transcription factor (Supplementary Table 6) and it was previously shown that sentinel IgG glycosylation SNP in the same region potentially affects binding of the IKZF1 transcription factor ${ }^{30}$. In addition, we observed protein-specific associations with two transcription factors: transferrin glycosylation was associated with variants in the HNF1A locus and IgG glycosylation was associated with variants in the IKZFI locus (Figure 1). We therefore checked expression of these genes in tissues where the two proteins are predominantly expressed. It is known that plasma transferrin, encoded by $T F$ gene, is mostly secreted by hepatocytes $^{48}$, while $\mathrm{IgG}$, the heavy chain constant region of which is encoded by $I G H G$ gene, is predominantly synthesised by the antibody-secreting plasma cells, the fully differentiated form of B-lymphocytes ${ }^{49}$. Indeed, we see that IGHG1 (encoding the most prevalent IgG1 subclass) is highly expressed in plasma cells and has low expression in hepatocytes, while the converse is true for $T F$ (Figure 5). Similarly, the transcription factor encoded by $H N F 1 A$ is predominantly expressed in the hepatocytes, while IKZFI is mainly expressed in plasma cells (Figure 5). Altogether these suggest that two distinct causal variants regulating glycosylation of transferrin and $\mathrm{IgG}$ in the FUT8 locus are likely to have tissuespecific effects, where the transferrin-associated variant affects the binding of HNF1A in liver and the IgG-associated variant affects the binding of IKZF1 in plasma cells, with both influencing expression of the FUT8 gene and therefore affecting fucosylation of the two proteins.

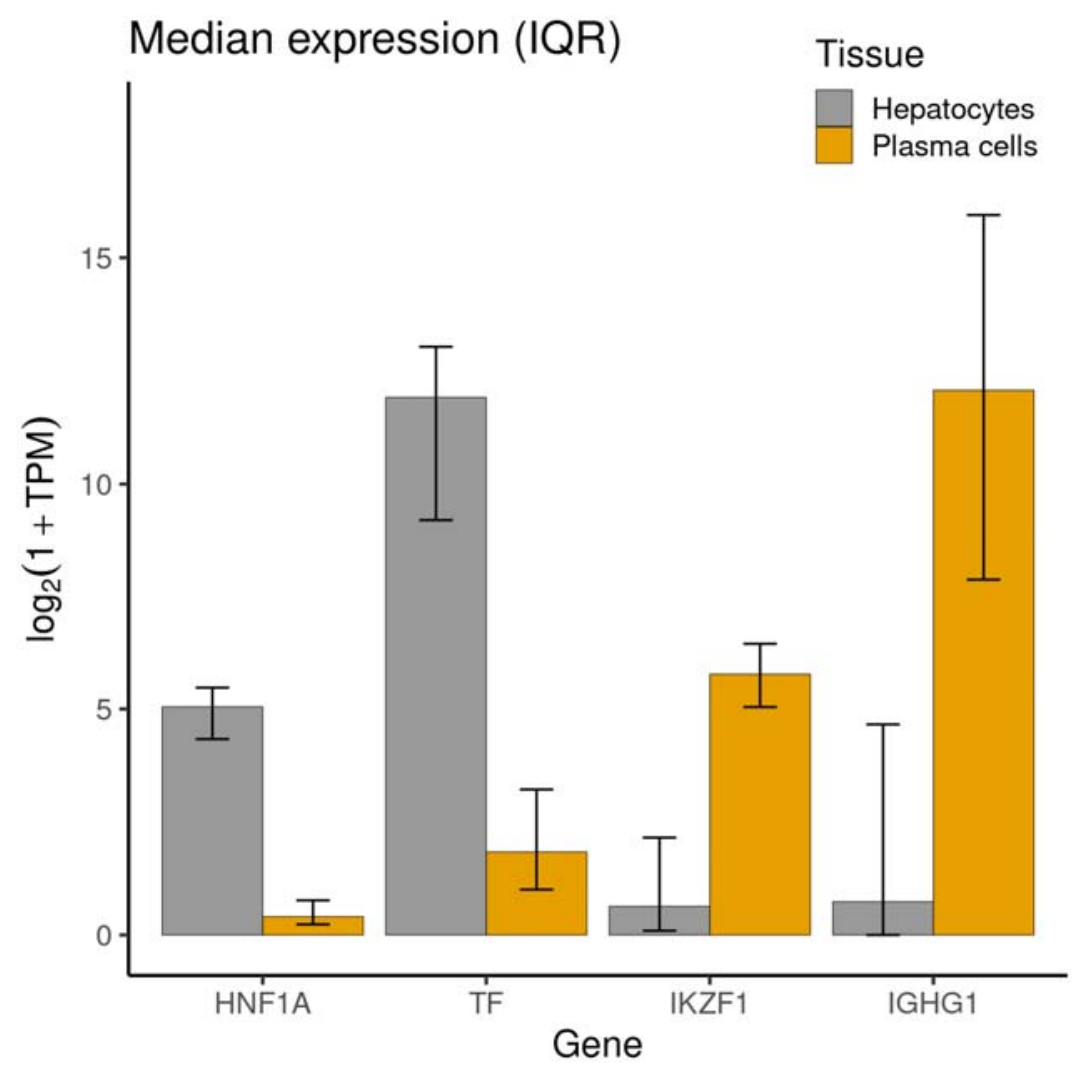

Figure 5. Expression of $H N F 1 A$ and $I K Z F 1$ in main tissues for transferrin and IgG proteins synthesis. $\log _{2}$ of median transcripts per million (TPM) and interquartile ranges are 
bioRxiv preprint doi: https://doi.org/10.1101/2021.05.04.442584; this version posted May 4, 2021. The copyright holder for this preprint (which was not certified by peer review) is the author/funder, who has granted bioRxiv a license to display the preprint in perpetuity. It is made available under aCC-BY-ND 4.0 International license.

reported for $T F$ (encoding transferrin protein), HNF1A, IGHG1 (encoding the constant region of immunoglobulin heavy chains) and IKZFI genes in plasma cells and hepatocytes. Gene expression data was obtained from the ARCHS4 portal $^{50}$. 


\section{$\underline{\text { Discussion }}$}

The post-translational modifications (PTMs) are essential mechanisms that dynamically regulate a large portion of cellular events by altering the structure and properties of proteins ${ }^{1}$. Similarly to other PTMs, genetic regulation of protein N-glycosylation has not been extensively investigated. Here, we performed genome-wide association meta-analysis of glycosylation of two proteins - transferrin and IgG - and compared how glycosylation of the two different proteins is genetically regulated. In the first GWAS of the transferrin $\mathrm{N}$ glycome, $(\mathrm{N}=1890)$, we identified 10 significantly associated loci, three of which (near $T F$, FOXII and MSRI) were never previously associated with the glycome of any protein. The other seven have been previously associated with glycosylation of total plasma proteins and/or IgG (Supplementary Table 2). The total plasma glycome quantifies the glycome of all proteins in plasma, but without information on which glycan was bound to which protein. Given that IgG and transferrin are among the most abundant plasma glycoproteins ${ }^{12}$, an overlap in genetic control of transferrin and IgG N-glycomes with that of total plasma proteins is to be expected. Sharapov et al. ${ }^{28}$ previously indicated that some of the genomic loci associated with the plasma glycome overlap with loci associated with $\operatorname{IgG} \mathrm{N}$ glycosylation. The present work suggests that the MGAT5, ST3GAL4, and B3GAT1 loci, that were also observed in the total plasma protein GWAS, might be capturing a signal within plasma protein glycosylation that comes mainly from transferrin $\mathrm{N}$-glycosylation.

We then compared the genetic architecture underlying glycosylation of transferrin and $\operatorname{IgG}$ proteins. Using the GWAS from this study we showed that there are both protein-specific and shared genetic loci. Looking specifically at glycosyltransferase enzymes, the main "drivers" of this post-translational modification, that catalyse the transfer of saccharide moieties from a donor to an acceptor molecule, MGAT5, ST3GALA, and B3GAT1 were only associated with transferrin while ST6GAL1 and MGAT3 were only associated with glycosylation of IgG. On the other hand, two fucosyltransferase genes, FUT8 and FUT6, were associated with both proteins. Even though the genes encoding these enzymes were associated with glycosylation of both proteins, using Approximate Bayes Factor colocalisation analysis, we showed that associations with transferrin and IgG $\mathrm{N}$-glycosylation at these genomic regions is driven by independent underlying causal variants, where one variant regulates fucosylation of transferrin and the other of IgG. Our results suggest that while the same fucosyltransferase enzymes are involved in $\mathrm{N}$-glycosylation of both transferrin and IgG proteins, the process is independently regulated by protein-specific causal variants.

There are at least two mechanisms that could explain how different variants in an enzymecoding gene could have distinct effects on two different substrates. If the two variants were in the coding region of the gene and affected the amino-acid sequence of the enzyme, they could affect the enzyme's specificity for binding each protein. However, none of the sentinel variants in the FUT8 and FUT6 loci were in strong linkage disequilibrium (LD) with coding variants from the enzymes' active sites, suggesting that this is likely not the mechanism of regulation of fucosylation of the two proteins. In addition, overall, SNPs associated with 
transferrin glycosylation predominantly mapped to regulatory rather than coding regions of the genome (Supplementary Table 5).

The other hypothesis is that these two variants affect the expression of enzymes in different tissues. In common with all other antibodies, most of IgG found in blood plasma is produced by bone marrow plasma cells, the fully differentiated form of B-cells ${ }^{49}$. The transferrin found in blood plasma is mostly produced by liver hepatocytes ${ }^{48}$. In addition, the glycomes of the two proteins were also associated with different transcription factor genes, namely, variants in $I K Z F 1$ region were associated with $I g G$ glycosylation, and variants in $H N F 1 A$ region with transferrin glycosylation. IKZF1, a transcription factor predominantly expressed in immune cells and tissues, has been functionally validated as a regulator of IgG core fucosylation: IKZF1 binds to regulatory regions of FUT8 and, in turn, knockdown of IKZF1 results in increased expression of FUT8 and increased core fucosylation of $\mathrm{IgG}^{30}$. On the other hand, we showed that transferrin glycosylation-associated variants in the FUT8 region might affect the binding of HNF1A, a transcription factor predominantly expressed in the liver. HNF1A has already been shown to regulate the expression of FUT8 and FUT6 and affects fucosylation of total plasma proteins ${ }^{27}$. Overall, we hypothesise that the two different causal variants affect the binding of different transcription factors in different tissues and therefore regulate the glycosylation of the two plasma proteins in a tissue-specific manner.

In addition to HNF1A, FUT8 glycosylation-associated variants might also be affecting the binding of the FOXI1 transcription factor. However, unlike HNF1A, possible involvement of FOXI1 in the regulation of the transferrin fucosylation is to date unknown and would require functional validation. We also found that HNF1A binding could also be affected by associated variants in the TF and ST3GAL4 genes. While these relationships were hitherto undocumented and need further supporting evidence, they may suggest that HNF1A might regulate multiple genes associated with transferrin $\mathrm{N}$-glycosylation.

Finally, our findings are not only relevant for unravelling the genetic mechanisms behind $\mathrm{N}$ glycosylation PTM but also contribute to understanding changes in N-glycan patterns involved in disease. The most strongly $\mathrm{N}$-glycosylation-associated variant for the $T F$ gene, rs6785596, was suggested by McClain et al. ${ }^{36}$ to regulate $T F$ expression in adipose tissue (also evident in GTEx v7) and consequently modulating insulin sensitivity. Excessive body iron stores represent a risk factor for decreased insulin sensitivity and diabetes ${ }^{51}$. McClain et al. ${ }^{36}$ argue that genetic downregulation of $T F$ expression in adipocytes has functional consequences for these cells' iron homeostasis and is sufficient to cause insulin resistance in humans and in a cell culture model. However, this SNP has so far not been associated with diabetes or diabetes-related traits, suggesting that this relationship needs to be explored further. Moreoever, while $T F$ variant rs6785596 is not associated with transferrin protein levels (pQTL), we can consider it as an example of a "cis-glyQTL": a genomic locus that explains variation in glycosylation levels and is local to the gene encoding the protein being glycosylated. Similar was observed for IgG glycosylation, where associated variants were mapping to the $I G H$ locus $^{32}$, a genetic region encoding the heavy chain of immunoglobulin $\mathrm{G}$. In addition, glycosylation SNPs in NXPE1/NXPE4 locus were pleiotropic with ulcerative colitis, a disease with abberant glycosylation patterns. 
In conclusion, by performing the first GWAS of the plasma transferrin $\mathrm{N}$-glycome and comparing it with that of the IgG $\mathrm{N}$-glycome, we were for the first time able to describe similarities and differences in the genetic regulation of post-translational modification of two different proteins. When focusing on glycosyltransferases, main enzymes of this PTM, we showed that there are both associations specific to each protein, but also those that are shared in glycosylation of the two proteins. For the shared associations, we showed that fucosylation of transferrin and IgG are regulated by independent, protein-specific variants in the FUT8 and FUT6 genes. In the FUT8 region these variants are likely to regulate fucosylation of transferrin and IgG in a tissue-specific manner, acting through tissue-specific transcription factors. Additional studies, with larger sample sizes and focusing on other non-IgG proteins, will be necessary to further unravel the genetic architecture of the N-glycosylation and other PTMs and to understand their relationship with human diseases and complex traits. 


\section{Materials and Methods}

\section{$\underline{\text { Population cohorts }}$}

The CROATIA-Korcula isolated population cohort includes samples of blood DNA, plasma and serum, anthropometric and physical measurements, information related to general health, medical history, lifestyle, and diet for $\sim 3000$ residents of the Croatian island of Korčula ${ }^{52}$. Written informed consent was given and the study was approved by the Ethics Committee of the Medical School, University of Split (approval id: 2181-198-03-04/10-11-0008). The Viking Health Study - Shetland (VIKING) is a family-based, cross-sectional study that seeks to identify genetic factors influencing cardiovascular and other disease risk in the population isolate of the Shetland Isles in northern Scotland ${ }^{53}$. Genetic diversity in this population is decreased compared to mainland Scotland, consistent with the high levels of endogamy. 2105 participants were recruited between 2013 and 2015, most having at least three grandparents from Shetland. Fasting blood samples were collected and many health-related phenotypes and environmental exposures were measured in each individual. All participants gave written informed consent and the study was approved by the South East Scotland Research Ethics Committee, NHS Lothian (reference: 12/SS/0151). Details of cohort-specific demographics, genotyping, quality control, and imputation performed before GWAS can be found in Supplementary Table 11 .

\section{Phenotypic data}

Transferrin and IgG N-glycome quantification. Transferrin and IgG N-glycome quantification for CROATIA-Korcula and VIKING samples was performed at Genos Glycobiology Laboratory. Isolation of the protein of interest and N-glycan quantification is described in more detail in Supplementary Materials and Methods for transferrin and by

Trbojević-Akmačić et al. ${ }^{54}$ for IgG. Briefly, proteins were first isolated from blood plasma (IgG depleted blood plasma in the case of transferrin) using affinity chromatography binding respectively to anti-transferrin antibodies plates for transferrin and protein $\mathrm{G}$ plates for IgG. The proteins isolation step was followed by enzymatic release and labelling of N-glycans with 2-AB (2-aminobenzamide) fluorescent dye. N-glycans were then separated and quantified by hydrophilic interaction ultra-high-performance liquid chromatography (HILICUHPLC). As a result, transferrin and IgG samples were separated into 35 (transferrin: TfGP1- TfGP35) and 24 (IgG: GP1-GP24) chromatographic peaks. It is worth noting that there is no correspondence structure-wise between transferrin TfGP and IgG GP traits labelled with the same number.

Normalisation and batch correction of glycan traits. Prior to genetic analysis, raw Nglycan UHPLC data was normalised and batch corrected to reduce the experimental variation in measurements. Total area normalisation was performed by dividing the area of each chromatographic peak (35 for transferrin, 24 for $\operatorname{IgG}$ ) by the total area of the corresponding chromatogram. Due to the multiplicative nature of measurement error and right-skewness of 
glycan data, normalised glycan measurements were log10-transformed. Batch correction was then performed using the empirical Bayes approach implemented in the "ComBat" function of the "sva" R package ${ }^{55}$, modelling the technical source of variation (96-well plate number) as batch covariate. Batch corrected measurements where then exponentiated back to the original scale.

\section{Genome-wide association analysis}

Genome-wide association analyses (GWAS) were performed in the two cohorts of European descent, CROATIA-Korcula and VIKING. Associations with 35 transferrin N-glycan traits were performed in 948 samples from CROATIA-Korcula and 959 samples from VIKING. Associations with $24 \mathrm{IgG}$ N-glycan traits were performed in 951 samples from CROATIAKorcula and 1086 samples from VIKING. The sample size of the same cohort differs between transferrin and IgG due to the different number of samples successfully measured for each protein. Prior to GWAS, each glycan trait was rank transformed to normal distribution using the "rntransform" function from the "GenABEL" R package 56 and then adjusted for age and sex, as fixed effects, and relatedness (estimated as the kinship matrix calculated from genotyped data) as random effect in a linear mixed model, calculated using the "polygenic" function from the "GenABEL" R package ${ }^{56}$. Residuals of covariate and relatedness correction were tested for association with HRC (Haplotype Reference Consortium) imputed SNP dosages using the RegScan v. 0.5 software $^{57}$, applying an additive genetic model of association.

\section{Meta-analysis}

Meta-analysis. Prior to meta-analysis the following quality control was performed on cohortlevel GWAS summary statistics. We removed all SNPs with a difference in allele frequency between the two cohorts higher than $+/-0.3$, as well as variants showing a minor allele count (MAC) lower or equal to 6. Cohort-level GWAS were then meta-analysed ( $\mathrm{N}=1890$ for transferrin and $\mathrm{N}=2020$ for IgG N-glycans, for $\sim 12$ million SNPs) using METAL software ${ }^{58}$, applying the fixed effect inverse-variance method, followed by genomic control correction. Mean genomic control inflation factor $\left(\lambda_{\mathrm{GC}}\right)$ was 0.997 (range 0.982-1.011) for transferrin $\mathrm{N}$ glycans and 0.995 (range 0.981-1.008) for IgG N-glycans meta-analysis, showing that the confounding effects of family structure were correctly accounted for.

Multiple test correction. The standard genome-wide significance threshold was Bonferroni corrected for the number of N-glycan traits analysed: variants were considered statistically significant if their $\mathrm{p}$-value was lower than $5 \times 10^{-8} / 35=1.43 \times 10^{-9}$ for transferrin and $5 \times 10^{-8} / 24=2.08 \times 10^{-9}$ for IgG N-glycan traits.

Locus definition. We used a positional approach to define genomic regions significantly associated with transferrin $\mathrm{N}$-glycan traits, following the procedure adopted by Sharapov et 
al. ${ }^{28}$ For each glycan trait, we grouped all genetic variants located within a $500 \mathrm{~kb}$ window $(+/-250 \mathrm{~kb})$ from the sentinel SNP in the same locus. To obtain a unique list of loci that are independent of the specific glycan trait, we then merged this list of sentinel SNP-glycan trait pairs for all 35 glycan traits and applied a similar procedure - all SNP-glycan trait pairs within a $1000 \mathrm{~kb}$ window (+/-500 kb from sentinel SNP) were grouped in the same locus, resulting in a unique list of sentinel SNP-top glycan trait pairs, summarising the genomic regions most strongly associated with $\mathrm{N}$-glycans across all traits. For all sentinel SNP-top glycan trait pairs, regional association plots were created with LocusZoom ${ }^{59}$ and visually checked - in case of overlapping patterns of association, only the sentinel SNP-top glycan trait pair showing the lowest $\mathrm{p}$-value was selected as a locus representative.

\section{$\underline{\text { Transferrin N-glycan traits post-meta-analysis follow-up }}$}

The meta-analysis follow-up analyses were performed only for the transferrin N-glycans meta-analysis, since genetic regulation of IgG N-glycosylation has already been explored in a larger, IgG-specific study ${ }^{30}$ and is beyond the scope of the present work.

Conditional analysis and phenotypic variance explained. To capture the overall contribution to phenotypic variation at each genomic region and identify secondary association signals at a locus, we performed approximate conditional analysis using the GCTA-COJO $^{39}$ stepwise model selection, "cojo-slct", with the transferrin N-glycan metaanalysis summary statistics and genotypes of 10,000 unrelated individuals of white British ancestry from UK Biobank ${ }^{60}$ as independent LD reference panel. Collinearity was restricted to 0.9 and the p-value threshold was set to $1.43 \times 10^{-9}$. Reported joint p-values were then adjusted by the genomic control method ${ }^{61}$. The list of samples for the independent LD reference panel was created with $\mathrm{R}$ 3.6.0, while the panel itself was generated using Plink $2.0^{62}$. After samples extraction from the UK Biobank full dataset, SNP deduplication was performed both by position (removing all SNPs not carrying a unique position on the chromosome) and marker name (--rm-dup exclude-all function). The proportion of variance in phenotype $(Y)$ explained by sentinel SNPs at each transferrin $\mathrm{N}$-glycans associated locus was calculated with the following formula

$$
\operatorname{Var}(Y)=\frac{2 \text { freq } *(1-\text { freq }) * \beta 2}{\operatorname{var}(Y \text { covariates adjustment residuals })}
$$

Gene prioritisation. For all genome-wide significant loci we suggested plausible candidate genes combining different evidence, namely evaluating biological role in the context of protein N-glycosylation of genes nearest to sentinel variants (positional mapping), assessing pleiotropy of sentinel variants with gene expression (expression quantitative trait loci, eQTL) or investigating associated variant's predicted effects on the protein sequence or on putative transcription factor binding sites (TFBSs). Positional gene mapping was performed using FUMA v1.3.5e SNP2GENE function ${ }^{63}$. Genes having a clear biological link to protein N- 
glycosylation (e.g. genes coding for enzymes involved in biochemical pathway of protein glycosylation) and genes previously associated with IgG and/or total blood plasma proteins $\mathrm{N}$-glycome were given a priority. The overlap of independent significant SNPs identified by COJO with eQTL was investigated using PhenoScanner v1.1 database ${ }^{45}$, taking into account significant genetic association ( $\mathrm{p}$-value $<5 \times 10^{-8}$ ) at the same or strongly ( $\mathrm{LD} \mathrm{r}^{2}>0.8$ ) linked SNPs in populations of European ancestry. The Ensembl Variant Effect Predictor (VEP v 97) tool $^{42}$ was used to determine putative functional effect and impact on a transcript or protein of independent significant SNPs and their strongly ( $\mathrm{LD} \mathrm{r}^{2}>0.8$ ) linked SNPs in populations of European ancestry. Among genes prioritised so far, two were transcription factors (i.e. HNF1A and FOXI1), while the remaining were non transcription factor protein-coding genes (i.e. MGAT5, TF, MSR1, NXPE1/NXPE4, ST3GAL4, B3GAT1, FUT8 and FUT6). Using the Regulatory sequence analysis tools (RSAT) program matrix-scan ${ }^{44}$, we applied a patternmatching procedure to search for sequences recognized as binding sites for HNF1A and FOXI1 transcription factors in associated regions of the other 8 prioritised genes. Positionspecific scoring matrices (PSSMs), representing the frequency of each nucleotide at each position of the transcription factor motif, were downloaded for HNF1A and FOXI1 from the $\mathrm{JASPAR}^{64}$ database. For each of the 8 genomic regions explored for possible transcription factor binding sites, we included the most strongly associated SNP and a $60 \mathrm{bp}$ surrounding sequence (30 bp either side of the sentinel SNP). The significance threshold was set to the pvalue $\leq 0.003$, Bonferroni corrected for 16 tests performed ( 8 putative transcription factor binding sites tested for 2 transcription factors).

Overlap and colocalization analysis with gene expression levels and complex traits. The PhenoScanner v1.1 database ${ }^{45}$ was used to investigate the overlap of significant transferrin glycosylation SNPs with gene expression levels and complex human traits. As previously described, we considered traits with genome-wide significant association ( $\mathrm{p}$-value $<5 \times 10^{-8}$ ) at the same or strongly (LD $\left.r^{2}>0.8\right)$ linked SNPs in populations of European ancestry. We then used Summary data-based Mendelian Randomization (SMR) analysis followed by the Heterogeneity in Dependent Instruments (HEIDI) test $^{41}$ to assess whether overlapping expression and complex traits, identified by PhenoScanner, were also colocalising with transferrin glycosylation (TfGP) traits. The SMR test indicates whether two traits are associated with the same locus, and HEIDI test specifies whether both traits are affected by the same underlying functional SNP. Each of 10 sentinel SNPs - TfGP pair (Table 1) was used for SMR/HEIDI analysis with gene expression levels and several complex traits. Summary statistics for gene expression levels in tissues/cell types were obtained from the Blood eQTL study ${ }^{65}$ (http://cnsgenomics.com/software/smr/\#eQTLsummarydata), the CEDAR project ${ }^{66}$ (http://cedar-web.giga.ulg.ac.be/), and the GTEx project version $7^{67}$ (https://gtexportal.org). Summary statistics for complex traits were obtained from various resources. In total, we used data for 3 tissues/cell types: CD19+ B lymphocytes (CEDAR), GTEx liver (GTEx) and peripheral blood (the Blood eQTL study) and 8 complex traits. Full list of GWAS collections, tissues and complex traits see in Supplementary Table 12. SMR/HEIDI analysis was performed according to the protocol described by Zhu et al. ${ }^{41} \mathrm{We}$ used sets of SNPs having the following properties: (1) being located within $\pm 250 \mathrm{~kb}$ from the sentinel SNPs identified in the present study; (2) being present in both the primary GWAS 
and eQTL data/GWAS for the complex trait; (3) having MAF $\geq 0.03$ in both datasets; (4) having squared Z-test value $\geq 10$ in the primary GWAS. Those SNPs that met criteria (1), (2), (3), (4), had the lowest P-value in the primary GWAS and were in high LD (r2 $\square>\square 0.8$ ) with the sentinel SNPs were used as instrumental variables to elucidate the relationship between gene expression/disease and TfGP (we define them as "top SNPs"). It should be noted that SMR/HEIDI analysis does not identify a causative SNP affecting both traits. It can be either the top SNP or any other SNP in strong LD. After defining the set of eligible SNPs for each locus, we made the "target" and "rejected" SNP sets and added the top SNP to the "target" set. Then we performed the following iterative procedure of SNP filtration: if the SNP from the eligible SNP set with the lowest PSMR had r2 >0.9 with any SNP in the "target" SNP set, it was added to the "rejected" set; otherwise, it was added to the "target" set. The procedure was repeated until eligible SNP set was exhausted, or the "target" set had 20 SNPs. If we were unable to select three or more SNPs, the HEIDI test was not conducted. HEIDI statistics was calculated as $T_{H E I D I}=\sum_{i}^{m} z_{d(i)}^{2}$, where $\mathrm{m}$ is the number of SNPs selected for analysis,

$$
\begin{gathered}
z_{d(i)}=d_{i} / S E_{\left(d_{i}\right)} \\
d_{i}=\beta_{S M R_{i}}-\beta_{S M R(t o p ~ S N P)}
\end{gathered}
$$

The results of the SMR test were considered statistically significant if PSMR $<1.7 \times 10^{-4}$ $(0.05 / 302$, where 302 is a total number of tests corresponding to analyzed loci and gene expression/disease traits). Inference of whether a functional variant may be shared between the TfGP and gene expression/disease were made based on the HEIDI test: $P_{\text {HEIDI }} \geq 0.001$ (possibly shared), and $\mathrm{P}_{\mathrm{HEIDI}}<0.001$ (sharing is unlikely).

\section{Colocalisation analysis for transferrin and IgG N-glycan traits}

The FUT8 and FUT6 genomic regions were significantly associated with both transferrin and IgG N-glycans. To investigate a possible overlap in genetic control of glycosylation between the two proteins, we used the approximate Bayes factor colocalisation analysis, developed by Giambartolomei et al. ${ }^{68}$ and implemented in "coloc" $\mathrm{R}$ package, followed by pairwise conditional and colocalization analysis $(\mathrm{PwCoCo})^{47}$ in case of multiple independent variants contributing to the trait variation. A posterior probability (PP) $>80 \%$ was considered as robust evidence supporting the tested hypothesis.

Overview of the overall procedure can be seen in Supplementary Figure 1. First, we assessed whether for one protein all glycans that are associated with the same genomic region ( $\mathrm{p}$-value $\leq 5 \times 10^{-8}$ ) are regulated by the same underlying variants. For each protein (i.e. transferrin and $\mathrm{IgG}$ ) and each genomic region (i.e. FUT8 and FUT6), we tested separately the group of glycans carrying only one independent association signal at locus and the group of glycan traits showing multiple independent signals of association (Supplementary figure 1). Pairs of glycan traits obtaining a PP.H4 > 80\% (suggestive of colocalisation) were pooled in the same colocalisation group, following the principle that if trait A colocalises with trait B and trait B 
colocalises with trait $\mathrm{C}$, thus also trait $\mathrm{A}$ and trait $\mathrm{C}$ colocalise. For each within-protein colocalisation group identified, the glycan trait with the lowest p-value was selected as group representative and carried on to the next step, where traits with single and multiple independent associations for each protein were tested for colocalisation. Similar to previous steps, glycan traits were grouped together on the basis of their colocalisation analysis results and the lowest p-value representative was chosen for the next step, where finally representative transferrin and IgG glycans were tested for between-protein colocalisation.

For glycan traits with multiple independent association signals and lacking strong evidence for colocalisation, we applied $\mathrm{PwCoCo}^{47}$ approach. Briefly, the PwCoCo approach tests not only the traits' full, complete GWAS association statistics for colocalisation, but also summary statistics conditioned for the top primary association, testing whether any of the underlying causal variants between traits colocalise. For example, assuming that each trait is carrying two conditionally independent association signals in the tested region, colocalisation analysis will be conducted between both full and conditioned association statistics (conditioned for each independent variable), for a total of nine pairwise combinations. Secondary association signals at FUT8 and FUT6 loci for both transferrin and IgG N-glycans were assessed using GCTA-COJO approximate conditional analysis stepwise model selection $^{39}$ and an LD reference panel of 10,000 unrelated, white British ancestry individuals from UK Biobank ${ }^{60}$. We then performed the association analysis conditional on identified secondary association signals at FUT8 and FUT6 loci using GCTA-COJO 39 "cojo-cond" and the same 10,000 UK Biobank samples LD reference panel, with $5 \times 10^{-8}$ p-value threshold and used those for pairwise colocalisation analyses.

Expression of $\mathrm{N}$-glycome associated genes in transferrin and IgG relevant tissues

Gene expression data for $T F, I G H G 1, H N F 1 A$ and $I K Z F 1$, expressed in gene counts, for hepatocytes (529 samples) and plasma cells (648 samples) was obtained from ARCHS4 portal $^{50}$. Samples with total number of gene counts less than 5,000,000 were filtered out. 513 hepatocyte and 53 plasma cell samples were undergone further analysis. Gene counts were scaled to transcripts per million (TPM) and $\log _{2}(1+\mathrm{TPM})$ transformed. 


\section{References}

1. Deribe, Y. L., Pawson, T. \& Dikic, I. Post-translational modifications in signal integration. Nature Structural and Molecular Biology 17, 666-672 (2010).

2. Choudhary, C. et al. Lysine acetylation targets protein complexes and co-regulates major cellular functions. Science (80-. ). 325, 834-840 (2009).

3. Santos, A. L. \& Lindner, A. B. Protein Posttranslational Modifications: Roles in Aging and Age-Related Disease. Oxidative Medicine and Cellular Longevity 2017, (2017).

4. Berdasco, M. \& Esteller, M. Aberrant Epigenetic Landscape in Cancer: How Cellular Identity Goes Awry. Developmental Cell 19, 698-711 (2010).

5. Leroy, G. et al. A quantitative atlas of histone modification signatures from human cancer cells. Epigenetics and Chromatin 6, (2013).

6. Mastrangelo, A. et al. The Role of Posttranslational Protein Modifications in Rheumatological Diseases: Focus on Rheumatoid Arthritis. Journal of Immunology Research 2015, (2015).

7. Mellor, K. M., Brimble, M. A. \& Delbridge, L. M. D. Glucose as an agent of posttranslational modification in diabetes - New cardiac epigenetic insights. Life Sciences 129, 48-53 (2015).

8. Miao, F. et al. Evaluating the role of epigenetic histone modifications in the metabolic memory of type 1 diabetes. Diabetes 63, 1748-1762 (2014).

9. Pena-Altamira, L., Polazzi, E. \& Monti, B. Histone post-translational modifications in Huntington's and Parkinson's diseases. Curr. Pharm. Des. 19, 5085-5092 (2013).

10. Santer, F. R. et al. Inhibition of the acetyltransferases p300 and CBP reveals a targetable function for $\mathrm{p} 300$ in the survival and invasion pathways of prostate cancer cell lines. Mol. Cancer Ther. 10, 1644-1655 (2011).

11. Lauc, G., Rudan, I., Campbell, H. \& Rudd, P. M. Complex genetic regulation of protein glycosylation. Molecular BioSystems 6, 329-335 (2010).

12. Clerc, F. et al. Human plasma protein N-glycosylation. Glycoconj. J. 33, 309-343 (2016).

13. Krištić, J. et al. Glycans are a novel biomarker of chronological and biological ages. Journals Gerontol. - Ser. A Biol. Sci. Med. Sci. 69, 779-789 (2014).

14. Russell, A. C. et al. The N-glycosylation of immunoglobulin $\mathrm{G}$ as a novel biomarker of Parkinson's disease. Glycobiology 27, 501-510 (2017).

15. Trbojević-Akmačić, I. et al. Plasma N-glycome composition associates with chronic low back pain. Biochim. Biophys. Acta - Gen. Subj. 1862, 2124-2133 (2018).

16. Gudelj, I. et al. Low galactosylation of $\mathrm{IgG}$ associates with higher risk for future diagnosis of rheumatoid arthritis during 10 $\square$ years of follow-up. Biochim. Biophys. 
Acta - Mol. Basis Dis. 1864, 2034-2039 (2018).

17. Trbojević-Akmačić, I. et al. Inflammatory bowel disease associates with proinflammatory potential of the immunoglobulin G glycome. Inflamm. Bowel Dis. 21, 1237-1247 (2015).

18. Rudman, N., Gornik, O. \& Lauc, G. Altered N-glycosylation profiles as potential biomarkers and drug targets in diabetes. FEBS Letters 593, 1598-1615 (2019).

19. Munkley, J. \& Elliott, D. J. Hallmarks of glycosylation in cancer. Oncotarget 7, 35478-89 (2016).

20. Taniguchi, N. \& Kizuka, Y. Glycans and Cancer: Role of N-Glycans in Cancer Biomarker, Progression and Metastasis, and Therapeutics. Adv. Cancer Res. 126, 1151 (2015).

21. Vajaria, B. N. \& Patel, P. S. Glycosylation: a hallmark of cancer? Glycoconjugate Journal 34, 147-156 (2017).

22. Rodriguez, E., Schetters, S. T. T. \& van Kooyk, Y. The tumour glyco-code as a novel immune checkpoint for immunotherapy. Nat. Rev. Immunol. 18, 204-211 (2018).

23. Adamczyk, B., Tharmalingam, T. \& Rudd, P. M. Glycans as cancer biomarkers. Biochim. Biophys. Acta - Gen. Subj. 1820, 1347-1353 (2012).

24. Peng, W. et al. Clinical application of quantitative glycomics. Expert Rev. Proteomics 15, 1007-1031 (2018).

25. Thanabalasingham, G. et al. Mutations in HNF1A result in marked alterations of plasma glycan profile. Diabetes 62, 1329-1337 (2013).

26. Huffman, J. E. et al. Polymorphisms in B3GAT1, SLC9A9 and MGAT5 are associated with variation within the human plasma N-glycome of 3533 European adults. Hum. Mol. Genet. 20, 5000-5011 (2011).

27. Lauc, G. et al. Genomics Meets Glycomics-The First GWAS Study of Human NGlycome Identifies HNF1 $\alpha$ as a Master Regulator of Plasma Protein Fucosylation. PLoS Genet. 6, e1001256 (2010).

28. Sharapov, S. Z. et al. Defining the genetic control of human blood plasma N-glycome using genome-wide association study. Hum. Mol. Genet. 28, 2062-2077 (2019).

29. Sharapov, S. Z. et al. Replication of 15 loci involved in human plasma protein Nglycosylation in 4802 samples from four cohorts. Glycobiology 31, 82-88 (2021).

30. Klarić, L. et al. Glycosylation of immunoglobulin $\mathrm{G}$ is regulated by a large network of genes pleiotropic with inflammatory diseases. Sci. Adv. 6, eaax0301 (2020).

31. Lauc, G. et al. Loci Associated with N-Glycosylation of Human Immunoglobulin G Show Pleiotropy with Autoimmune Diseases and Haematological Cancers. PLoS Genet. 9, e1003225 (2013). 
32. Shen, X. et al. Multivariate discovery and replication of five novel loci associated with Immunoglobulin G N-glycosylation. Nat. Commun. 8, 447 (2017).

33. Wahl, A. et al. Genome-wide association study on immunoglobulin G glycosylation patterns. Front. Immunol. 9, 277 (2018).

34. Li, T. et al. Modulating IgG effector function by Fc glycan engineering. Proc. Natl. Acad. Sci. U. S. A. 114, 3485-3490 (2017).

35. Mimura, Y. et al. Glycosylation engineering of therapeutic IgG antibodies: challenges for the safety, functionality and efficacy. Protein and Cell 9, 47-62 (2018).

36. McClain, D. A. et al. Adipose tissue transferrin and insulin resistance. J. Clin. Endocrinol. Metab. 103, 4197-4208 (2018).

37. Karlsson, I., Ndreu, L., Quaranta, A. \& Thorsén, G. Glycosylation patterns of selected proteins in individual serum and cerebrospinal fluid samples. J. Pharm. Biomed. Anal. 145, 431-439 (2017).

38. Spik, G. et al. Studies on glycoconjugates. LXIV. Complete structure of two carbohydrate units of human serotransferrin. FEBS Lett. 50, 296-299 (1975).

39. Yang, J. et al. Conditional and joint multiple-SNP analysis of GWAS summary statistics identifies additional variants influencing complex traits. Nat. Genet. 44, 369375 (2012).

40. Wahl, A. et al. IgG glycosylation and DNA methylation are interconnected with smoking. Biochim. Biophys. Acta - Gen. Subj. 1862, 637-648 (2018).

41. Zhu, Z. et al. Integration of summary data from GWAS and eQTL studies predicts complex trait gene targets. Nat. Genet. 48, 481-487 (2016).

42. McLaren, W. et al. The Ensembl Variant Effect Predictor. Genome Biol. 17, 122 (2016).

43. Mollicone, R. et al. Molecular basis for plasma $\alpha(1,3)$-fucosyltransferase gene deficiency (FUT6). J. Biol. Chem. 269, 12662-12671 (1994).

44. Turatsinze, J. V., Thomas-Chollier, M., Defrance, M. \& van Helden, J. Using RSAT to scan genome sequences for transcription factor binding sites and cis-regulatory modules. Nat. Protoc. 3, 1578-1588 (2008).

45. Staley, J. R. et al. PhenoScanner: A database of human genotype-phenotype associations. Bioinformatics 32, 3207-3209 (2016).

46. Martensson, O., Harlin, A., Brandt, R., Seppa, K. \& Sillanaukee, P. Transferrin Isoform Distribution: Gender and Alcohol Consumption. Alcohol. Clin. Exp. Res. 21, 1710-1715 (1997).

47. Zheng, J. et al. Phenome-wide Mendelian randomization mapping the influence of the plasma proteome on complex diseases. Nat. Genet. 52, 1122-1131 (2020). 
48. Ogun, A. S. \& Adeyinka, A. Biochemistry, Transferrin. StatPearls (2021).

49. Allen, H. C. \& Sharma, P. Histology, Plasma Cells. in StatPearls (2021).

50. Lachmann, A. et al. Massive mining of publicly available RNA-seq data from human and mouse. Nat. Commun. 9, 1-10 (2018).

51. Jiang, R. et al. Body Iron Stores in Relation to Risk of Type 2 Diabetes in Apparently Healthy Women. J. Am. Med. Assoc. 291, 711-717 (2004).

52. Zemunik, T. et al. Genome-wide association study of biochemical traits in Korcula Island, Croatia. Croat. Med. J. 50, 23-33 (2009).

53. Kerr, S. M. et al. An actionable KCNH2 Long QT Syndrome variant detected by sequence and haplotype analysis in a population research cohort. Sci. Rep. 9, (2019).

54. Trbojević Akmačić, I. et al. High-throughput glycomics: Optimization of sample preparation. Biochem. 80, 934-942 (2015).

55. Johnson, W. E., Li, C. \& Rabinovic, A. Adjusting batch effects in microarray expression data using empirical Bayes methods. Biostatistics 8, 118-127 (2007).

56. Karssen, L. C., van Duijn, C. M. \& Aulchenko, Y. S. The GenABEL Project for statistical genomics. F1000Research 5, (2016).

57. Haller, T., Kals, M., Esko, T., Mägi, R. \& Fischer, K. RegScan: A GWAS tool for quick estimation of allele effects on continuous traits and their combinations. Brief. Bioinform. 16, 39-44 (2013).

58. Willer, C. J., Li, Y. \& Abecasis, G. R. METAL: Fast and efficient meta-analysis of genomewide association scans. Bioinformatics 26, 2190-2191 (2010).

59. Pruim, R. J. et al. LocusZoom: Regional visualization of genome-wide association scan results. in Bioinformatics 27, 2336-2337 (2011).

60. Bycroft, C. et al. The UK Biobank resource with deep phenotyping and genomic data. Nature 562, 203-209 (2018).

61. Devlin, B. \& Roeder, K. Genomic control for association studies. Biometrics 55, 9971004 (1999).

62. Chang, C. C. et al. Second-generation PLINK: Rising to the challenge of larger and richer datasets. Gigascience 4, 7 (2015).

63. Watanabe, K., Taskesen, E., Van Bochoven, A. \& Posthuma, D. Functional mapping and annotation of genetic associations with FUMA. Nat. Commun. 8, 1-11 (2017).

64. Vlieghe, D. et al. A new generation of JASPAR, the open-access repository for transcription factor binding site profiles. Nucleic Acids Res. 34, D95-D97 (2006).

65. Westra, H. J. et al. Systematic identification of trans eQTLs as putative drivers of known disease associations. Nat. Genet. 45, 1238-1243 (2013). 
66. Momozawa, Y. et al. IBD risk loci are enriched in multigenic regulatory modules encompassing putative causative genes. Nat. Commun. 9, 1-18 (2018).

67. Lonsdale, J. et al. The Genotype-Tissue Expression (GTEx) project. Nature Genetics 45, 580-585 (2013).

68. Giambartolomei, C. et al. Bayesian Test for Colocalisation between Pairs of Genetic Association Studies Using Summary Statistics. PLoS Genet. 10, (2014). 


\section{Acknowledgements}

The CROATIA_Korcula study was funded by grants from the MRC (United Kingdom), European Commission Framework 6 project EUROSPAN (contract number LSHG-CT-2006018947), Croatian Science Foundation (grant 8875), and the Republic of Croatia Ministry of Science, Education and Sports (216-1080315-0302). Genotyping was performed in the Genetics Core of the Clinical Research Facility, University of Edinburgh. We would like to acknowledge all the staff of several institutions in Croatia that supported the CROATIA_ Korcula fieldwork, including, but not limited to, the University of Split and Zagreb Medical Schools, Institute for Anthropological Research in Zagreb, and the Croatian Institute for Public Health in Split. The Viking Health Study - Shetland (VIKING) was supported by the MRC Human Genetics Unit quinquennial programme grant "QTL in Health and Disease". DNA extractions and genotyping were performed at the Edinburgh Clinical Research Facility, University of Edinburgh. We would like to acknowledge the invaluable contributions of the research nurses in Shetland, the administrative team in Edinburgh and the people of Shetland. We acknowledge support from the MRC Human Genetics Unit programme grant, "Quantitative traits in health and disease" (U. MC_UU_00007/10).

A.L. was funded by the European Union's Horizon 2020 research and innovation program IMforFUTURE, under H2020-MSCA-ITN grant agreement number 721815. The work of L.K. was supported by an RCUK Innovation Fellowship from the National Productivity Investment Fund (MR/R026408/1). The work of Y.S.A. was supported by a grant from the Russian Science Foundation (RSF) No. 19-15-00115. The work of J.F.W. and C.H. was supported by an MRC University Unit Programme Grant MC_UU_00007/10 "QTL in Health and Disease".

\section{Author contributions}

A.L.: Data analysis and interpretation, Visualization, Writing-Original draft preparation, Writing-Review and editing. I.T.-A.: Quantification of transferrin and IgG N-glycans, Data interpretation, Writing-Original draft preparation, Writing-Review and editing. P.N.: Supervision, Data interpretation, Writing-Review and editing. Y.T.: Data analysis and interpretation, Writing-Original draft preparation. S.Z.S.: Visualization, Writing-Original draft preparation. F.V.: Glycan data quality control. O.P.: Genomic and demographic data provider for CROATIA-Korcula cohort. C.H.: Genomic and demographic data provider for CROATIA-Korcula cohort. T.P.: Quantification of transferrin and IgG N-glycans. M.V.: Quantification of transferrin and IgG N-glycans. Y.S.A.: Writing-Review and editing. G.L.: Glycan data provider for CROATIA-Korcula and VIKING cohorts, Writing-Review and editing. J.F.W.: Conceptualization, Genomic and demographic data provider for VIKING cohort, Supervision, Data interpretation, Writing-Original draft preparation, WritingReview and editing. L.K.: Conceptualization, Supervision, Data interpretation, WritingOriginal draft preparation, Writing-Review and editing. 


\section{Competing interests}

G.L. is the founder and owner of Genos Ltd, a private research organization that specializes in high-throughput glycomic analysis and has several patents in this field. I.T.-A., F.V., T.P., and M.V. are employees of Genos Ltd. Y.S.A. is a founder and a co-owner of PolyOmica and PolyKnomics, private organizations providing services, research and development in the field of computational and statistical genomics. 